\title{
ON THE RELATION BETWEEN MOORE'S AND PENROSE'S CONDITIONS
}

\author{
GAOXIONG GAN
}

Received 13 April 2001

\begin{abstract}
Moore (1920) defined the reciprocal of any matrix over the complex field by three conditions, but the beauty of the definition was not realized until Penrose (1955) defined the same inverse using four conditions. The reciprocal is now often called the MoorePenrose inverse, and has been widely used in various areas. This note comments on the definitions of Moore-Penrose inverse, and gives a new characterization for two types of weak Moore-Penrose inverses, which exposes an important relation between Moore's and Penrose's conditions. It also attempts to emphasize the merit of Moore's definition, which has been overlooked mainly due to Moore's unique notation. Two examples are given to demonstrate some combined applications of Moore's and Penrose's conditions, including a correction for an incorrect proof of Ben-Israel's (1986) characterization for Moore's conditions.
\end{abstract}

2000 Mathematics Subject Classification: 15A09.

1. Introduction. Denote by $\mathscr{C}^{m \times n}$ the set of $m \times n$ finite matrices over the field of complex numbers, and by $A^{*}$ the conjugate transpose of a $\mathscr{C}^{m \times n}$-matrix $A$. Moore [4] defined the reciprocal $\Lambda$ of a $\mathscr{C}^{m \times n}$-matrix $K$ as a $\mathscr{C}^{n \times m}$-matrix such that (a) the columns of $\Lambda$ are linear combinations of the conjugates of the rows of $K$, (b) the rows of $\Lambda$ are linear combinations of the conjugates of the columns of $K$, (c) the matrix $K \Lambda K$ obtained by matricial composition of the matrices $K, \Lambda, K$ is the original matrix $K$. In other words, $\Lambda$ satisfies the following three conditions:

(a) $\Lambda=K^{*} C$ for some $C \in \mathscr{C}^{m \times m}$,

(b) $\Lambda=D K^{*}$ for some $D \in \mathscr{C}^{n \times n}$,

(c) $K \Lambda K=K$.

Alternatively, if we denote by $\operatorname{Col}(K)$ the column space of $K$, Moore's conditions (a) and (b) can be written as (a) $\operatorname{Col}(\Lambda) \subset \operatorname{Col}\left(K^{*}\right)$ and (b) $\operatorname{Col}\left(\Lambda^{*}\right) \subset \operatorname{Col}(K)$. Furthermore, the existence and uniqueness of $\Lambda$ were announced in [4], and proven in [5, pages 8 , 202] after Moore's death, where the reciprocal was renamed as the general reciprocal.

However, the beauty of Moore's definition was not realized until Penrose [7] defined the generalized inverse $A^{\dagger}$ of a matrix $A \in \mathscr{C}^{m \times n}$ as the solution of the following four equations:

(1) $A A^{\dagger} A=A$,

(2) $A^{\dagger} A A^{\dagger}=A^{\dagger}$,

(3) $A A^{\dagger}=\left(A A^{\dagger}\right)^{*}$,

(4) $A^{\dagger} A=\left(A^{\dagger} A\right)^{*}$,

and proved the existence and uniqueness of $A^{\dagger}$. Moore's publications were not cited 
in Penrose [7]. Since then the $A^{\dagger}$ has been widely used in various areas, especially in the study of approximate solutions (such as the least-squares solutions) of linear equations.

The equivalence between Moore's reciprocal and Penrose's generalized inverse was shown by Rado [10], and Penrose [8] noticed this too. The $A^{\dagger}$ is now often called the Moore-Penrose inverse ( $M$-P inverse) and sometimes is also called the pseudo-inverse. We denote by $A^{\dagger}$ the M-P inverse of $A$.

Another equivalent definition of the M-P inverse, often used in algebraic textbooks, is as follows: if $A$ is of rank $r,\left\{b_{1}, \ldots, b_{r}\right\}$ is a basis of $\operatorname{Col}(A)$, and $B:=\left[b_{1}, \ldots, b_{r}\right]$, (hence $A=B H$ for some $H \in \mathscr{C}^{r \times n}$ of rank $r$ ), then

$$
A^{\dagger}=H^{*}\left(H H^{*}\right)^{-1}\left(B^{*} B\right)^{-1} B^{*} \quad \text { or } \quad A^{\dagger}=H^{*}\left(B^{*} A H^{*}\right)^{-1} B^{*}
$$

(see Ben-Israel and Greville [2, page 23]). The existence of $A^{\dagger}$ follows from this definition immediately; and if $A$ is of full-rank, an algorithm for computing $A^{\dagger}$ follows from the definition as well (see Noble [6, page 291]). But this definition does not describe the features of $A^{\dagger}$ explicitly, while Moore's and Penrose's definitions do.

The weak M-P inverses (or weak generalized inverses) are also playing an important role in applications. We denote by $A^{\left\{i_{1}, \ldots, i_{k}\right\}}$ a weak M-P inverse of $A$ satisfying Moore's or Penrose's conditions $\left(i_{1}\right), \ldots,\left(i_{k}\right)$ (hence $A^{\dagger} \equiv A^{\{a, b, c\}} \equiv A^{\{1,2,3,4\}}$ and $A^{\{c\}} \equiv A^{\{1\}}$ ), and by $\mathscr{A}^{\left\{i_{1}, \ldots, i_{k}\right\}}$ the set of all possible $A^{\left\{i_{1}, \ldots, i_{k}\right\}}$ 's (hence $\mathscr{A}^{\left\{i_{1}, \ldots, i_{k}\right\}} \subset \mathscr{C}^{n \times m}, \mathscr{A}^{\{1,2,3,4\}}=$ $\mathscr{A}^{\{a, b, c\}}=\left\{A^{\dagger}\right\}$, and $\mathscr{A}^{\{c\}} \equiv \mathscr{A}^{\{1\}}$ ).

In addition to the comments on the definitions of M-P inverse, this note gives a new characterization for two types of weak M-P inverses, namely $\mathscr{A}^{\{a, c\}}=\mathscr{A}^{\{1,2,4\}}$ and $\mathscr{A}^{\{b, c\}}=\mathscr{A}^{\{1,2,3\}}$, which exposes an important relation between Moore's and Penrose's conditions. It also attempts to emphasize the merit of Moore's definition, which has been overlooked mainly due to Moore's unique notation. Two examples are given to demonstrate some combined applications of Moore's and Penrose's conditions, including a correction for an incorrect proof of Ben-Israel's [1] characterization for Moore's conditions (a) and (b).

2. Characterization for two types of weak M-P inverses. Ben-Israel [1] examined Penrose's $A^{\dagger}$ in relation to Moore's $A^{\{a, b, c\}}$, interpreted Moore's results in a more readable manner, and gave (as [1, Lemma 1]) a characterization of $\mathscr{A}^{\{a, b\}}$ that

$$
\mathscr{A}^{\{a, b\}}=\left\{X=A^{*} U A^{*}: U \in \mathscr{C}^{m \times n}\right\}
$$

supported by an incorrect proof, which is corrected in Example 3.2. Pringle and Rayner [9, page 26] characterized weak M-P inverses $A^{\{1,2,3\}}$ and $A^{\{1,2,4\}}$, using a result of Goldman and Zelen [3], and showed over the real field that for an $m \times n$ matrix $A$,

$$
G \text { is an } A^{\{1,2,3\}} \text { iff } G=\left(A^{\prime} A\right)^{\{1\}} A^{\prime} \text { for some }\left(A^{\prime} A\right)^{\{1\}} \text {, }
$$

where $A^{\prime}$ denotes the transpose of $A$; and

$$
G \text { is an } A^{\{1,2,4\}} \text { iff } G=A^{\prime}\left(A A^{\prime}\right)^{\{1\}} \text { for some }\left(A A^{\prime}\right)^{\{1\}} \text {. }
$$

Plainly, (2.2) and (2.3) hold over the complex field. 
In what follows, $A$ denotes a $\mathscr{C}^{m \times n}$-matrix.

Also note that for any subsets $X$ and $Y$ of $\{a, b, c, 1,2,3,4\}$,

$$
\mathscr{A}^{X \cup Y}=\mathscr{A}^{X} \cap \mathscr{A}^{Y} \quad \text { with } \mathscr{A}^{\varnothing}:=\mathscr{C}^{n \times m}
$$

(hence $\left.\mathscr{A}^{X} \subset \mathscr{C}^{n \times m}\right)$.

THEOREM 2.1. It is true that $\mathscr{A}^{\{a, c\}}=\mathscr{A}^{\{1,2,4\}}$ and $\mathscr{A}^{\{b, c\}}=\mathscr{A}^{\{1,2,3\}}$.

Proof. For $G \in \mathscr{A}^{\{a, c\}}$, there exists $C \in \mathscr{C}^{m \times m}$ such that

$$
G^{*}=C^{*} A=C^{*} A G A=G^{*} G A,
$$

so $(G A)^{*}=A^{*} G^{*} G A=G A$, and hence $G \in \mathscr{A}^{\{1,4\}}$. It then follows that

$$
G^{*}=G^{*} G A=G^{*} A^{*} G^{*},
$$

hence $\mathscr{A}^{\{a, c\}} \subset \mathscr{A}^{\{1,2,4\}}$. Conversely, for $G \in \mathscr{A}^{\{1,2,4\}}$,

$$
G=G A G=A^{*} G^{*} G
$$

hence $\mathscr{A}^{\{1,2,4\}} \subset \mathscr{A}^{\{a, c\}}$.

Next, for $G \in \mathscr{A}^{\{b, c\}}$, there exists $D \in \mathscr{C}^{n \times n}$ such that

$$
G=D A^{*}=D A^{*} G^{*} A^{*}=G G^{*} A^{*},
$$

so $A G=A G G^{*} A^{*}=(A G)^{*}$, hence $G \in \mathscr{A}^{\{1,3\}}$. It then follows that

$$
G=G G^{*} A^{*}=G A G
$$

hence $\mathscr{A}^{\{b, c\}} \subset \mathscr{A}^{\{1,2,3\}}$. Conversely, for $G \in \mathscr{A}^{\{1,2,3\}}$,

$$
G=G A G=G G^{*} A^{*},
$$

hence $\mathscr{A}^{\{1,2,3\}} \subset \mathscr{A}^{\{b, c\}}$.

Theorem 2.1 exposes an important relation between Moore's and Penrose's conditions that one of Moore's conditions (a) and (b) plus the common condition (c) is equivalent to Penrose's conditions (1) and (2) plus one of conditions (3) and (4). This is also a new characterization for two types, $A^{\{1,2,3\}}$ and $A^{\{1,2,4\}}$, of weak M-P inverses. Combining Theorem 2.1 with the results (2.2) and (2.3) of Pringle and Rayner [9], we get

$$
\begin{aligned}
& G \in A^{\{1,2,4\}} \text { iff } G \in A^{\{a, c\}} \text { iff } G=A^{*}\left(A A^{*}\right)^{\{c\}} \text { for some }\left(A A^{*}\right)^{\{c\}}, \\
& G \in A^{\{1,2,3\}} \text { iff } G \in A^{\{b, c\}} \text { iff } G=\left(A^{*} A\right)^{\{c\}} A^{*} \text { for some }\left(A^{*} A\right)^{\{c\}} .
\end{aligned}
$$

Rado's [10] proof for the equivalence between Moore's $A^{\{a, b, c\}}$ and Penrose's $A^{\{1,2,3,4\}}$ is somewhat tedious. Now the equivalence, $\mathscr{A}^{\{1,2,3,4\}}=\mathscr{A}^{\{a, b, c\}}$, can be easily shown via Theorem 2.1 and (2.4) as follows:

$$
\mathscr{A}^{\{a, b, c\}}=\mathscr{A}^{\{a, c\}} \cap \mathscr{A}^{\{b, c\}}=\mathscr{A}^{\{1,2,4\}} \cap \mathscr{A}^{\{1,2,3\}}=\mathscr{A}^{\{1,2,3,4\}} .
$$


3. Comment and examples. Most research publications in the area of generalized inversion adopt Penrose's definition of four conditions, and overlook Moore's definition of three conditions. If Moore's unique notation was a reason for this phenomenon (see Ben-Israel [1], Pringle and Rayner [9, page 3]), it seems that we should pay more attention to Moore's definition now.

In fact, Moore's conditions (a) and (b) are quite intuitive and convenient to use.

In some cases, combined applications of Moore's and Penrose's conditions can simplify approaches to a great extent.

EXAMPLE 3.1. The results (2.2) and (2.3) of Pringle and Rayner [9] can be easily proved (over the complex field) as follows: for (2.2), if $G=\left(A^{*} A\right)^{\{1\}} A^{*}$ for some $\left(A^{*} A\right)^{\{1\}}$, then $G \in \mathscr{A}^{\{b\}}$, and

$$
A G A=A^{\{1,3\}^{*}} A^{*} A\left(A^{*} A\right)^{\{1\}} A^{*} A=A,
$$

so $G \in \mathscr{A}^{\{b, c\}}$. By Theorem 2.1, $G \in \mathscr{A}^{\{1,2,3\}}$. Conversely, if $G \in \mathscr{A}^{\{1,2,3\}}$, by Theorem 2.1, $G \in \mathscr{A}^{\{b, c\}}$. Choose $D \in \mathscr{C}^{n \times n}$ such that $G=D A^{*}$, then

$$
A^{*} A D A^{*} A=A^{*} A G A=A^{*} A .
$$

Thus, $D$ is an $\left(A^{*} A\right)^{\{1\}}$ and $G=\left(A^{*} A\right)^{\{1\}} A^{*}$.

The dual result (2.3) is proved in a similar manner.

EXAMPLE 3.2. Equation (2.1) is given by Ben-Israel's [1, Lemma 1], and is true, but the proof in [1] is incorrect. To point out the incorrectness, we copy (with minor wording modification) the main part of the proof: if $X=Y A^{*}=A^{*} Z$ for some $Y$ and $Z$, and define $Y_{1}, Y_{2}, Z_{1}, Z_{2}$ by

$$
Y=A^{*} Y_{1}+Y_{2}, \quad A Y_{2}=0, \quad Z=Z_{1} A^{*}+Z_{2}, \quad Z_{2} A=0,
$$

then $A X A=A A^{*} Y_{1} A^{*} A=A A^{*} Z_{1} A^{*} A$, proving that $Y_{1}=Z_{1}, \ldots$

Plainly, the consequence, $Y_{1}=Z_{1}$, need not be true.

Indeed, (2.1) can be proved as follows: for $X \in \mathscr{A}^{\{a, b\}}$, choose $Y \in \mathscr{C}^{n \times n}$ and $Z \in$ $\mathfrak{C}^{m \times m}$ such that $X=Y A^{*}=A^{*} Z$. Then

$$
X=Y A^{*}=Y A^{*} A^{*\{c\}} A^{*}=A^{*} Z A^{*\{c\}} A^{*} \quad \text { with } U=Z A^{*\{c\}} .
$$

The converse has nothing to prove.

Note 3.3. To prove (2.1), there are many alternative choices of $U$, such as $U=$ $A^{*\{c\}} Y$ or $A^{*\{c\}} X A^{*\{c\}}$.

\section{REFERENCES}

[1] A. Ben-Israel, Generalized inverses of matrices: a perspective of the work of Penrose, Math. Proc. Cambridge Philos. Soc. 100 (1986), no. 3, 407-425.

[2] A. Ben-Israel and T. N. E. Greville, Generalized Inverses: Theory and Applications, WileyInterscience, New York, 1974.

[3] A. J. Goldman and M. Zelen, Weak generalized inverses and minimum variance linear unbiased estimation, J. Res. Nat. Bur. Standards Sect. B 68B (1964), 151-172. 
[4] E. H. Moore, On the reciprocal of the general algebraic matrix, Bull. Amer. Math. Soc. 26 (1920), 394-395.

[5] _ General Analysis. Part I. The Algebra of Matrices, Memoirs of the American Philosophical Society, vol. 1, American Philosophical Society, Pennsylvania, 1935.

[6] B. Noble, Methods for computing the Moore-Penrose generalized inverse, and related matters, Generalized Inverses and Applications (Proc. Sem., Math. Res. Center, Univ. Wisconsin, Madison, Wis., 1973), Publ. Math. Res. Center Univ. Wisconsin, no. 32, Academic Press, New York, 1976, pp. 245-301.

[7] R. Penrose, A generalized inverse for matrices, Math. Proc. Cambridge Philos. Soc. 51 (1955), 406-413.

[8]___ On best approximation solutions of linear matrix equations, Math. Proc. Cambridge Philos. Soc. 52 (1956), 17-19.

[9] R. M. Pringle and A. A. Rayner, Generalized Inverse Matrices with Applications to Statistics, Griffin's Statistical Monographs and Courses, no. 28, Hafner Publishing, New York, 1971.

[10] R. Rado, Note on generalized inverses of matrices, Math. Proc. Cambridge Philos. Soc. 52 (1956), 600-601.

GAOXIONG GAN: DEPARTMENT OF MATHEMATICS AND STATISTICS, UNIVERSITY OF MisSOURIROLLA, 1870 MiNER CiRCLE, ROLLA, MO 65409-0020, USA 


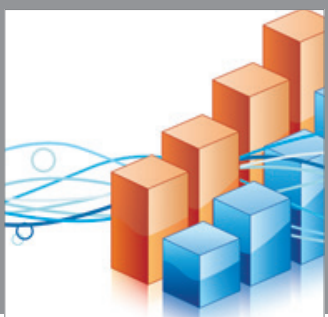

Advances in

Operations Research

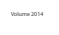

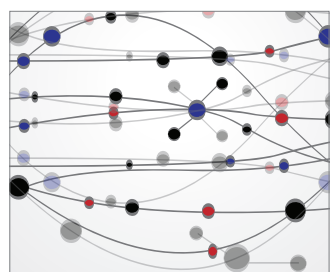

\section{The Scientific} World Journal
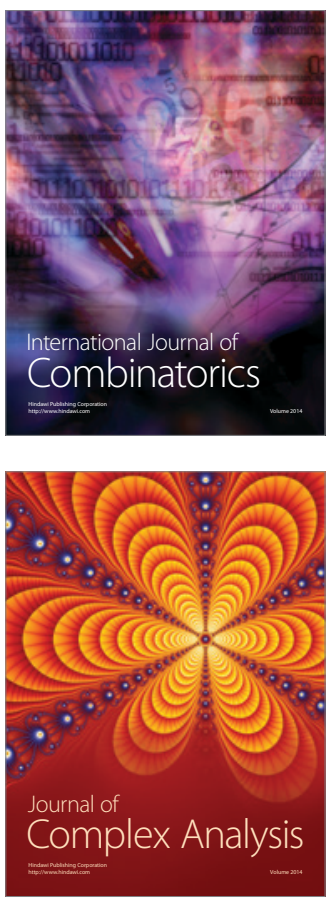

International Journal of

Mathematics and

Mathematical

Sciences
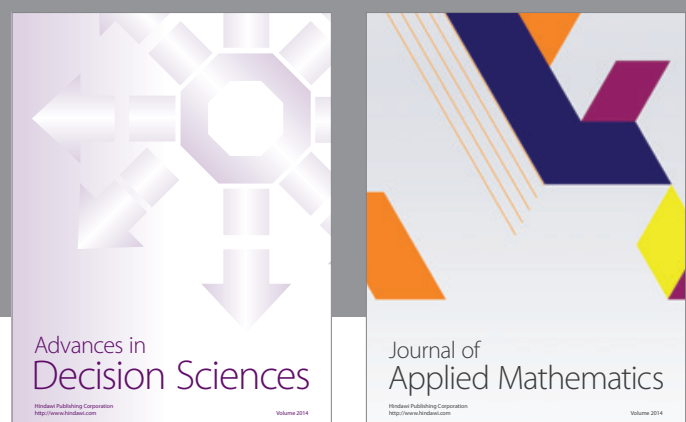

Journal of

Applied Mathematics
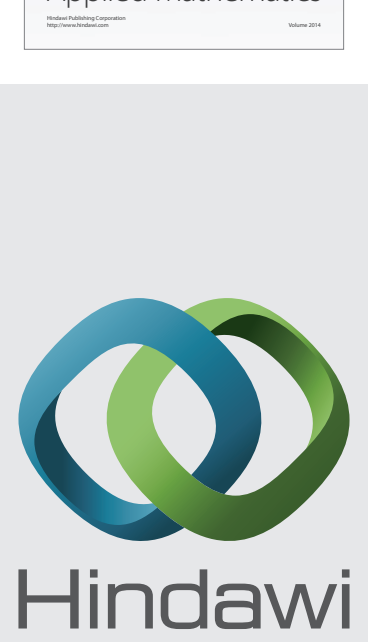

Submit your manuscripts at http://www.hindawi.com
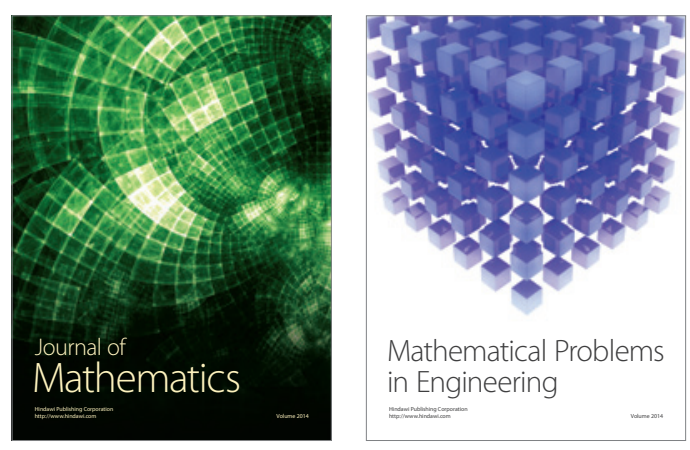

Mathematical Problems in Engineering
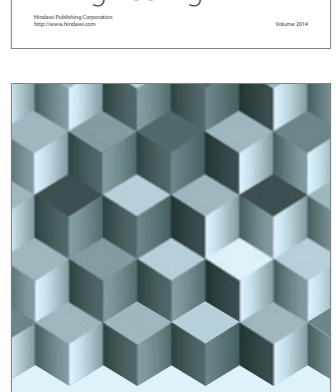

Journal of

Function Spaces
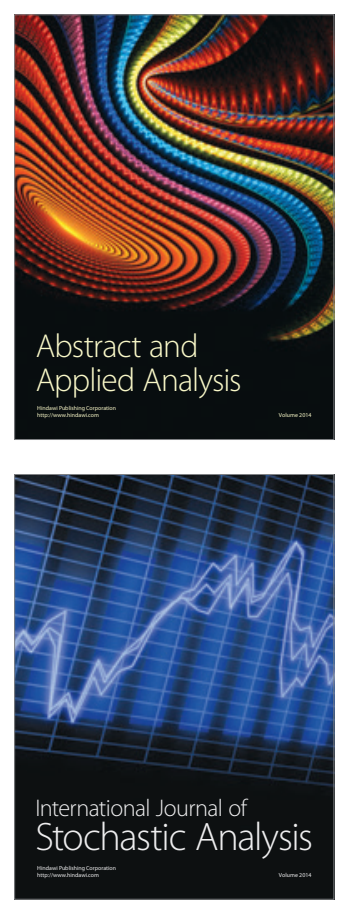

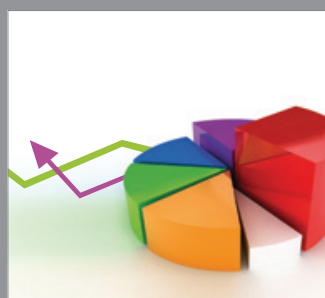

ournal of

Probability and Statistics

Promensencen
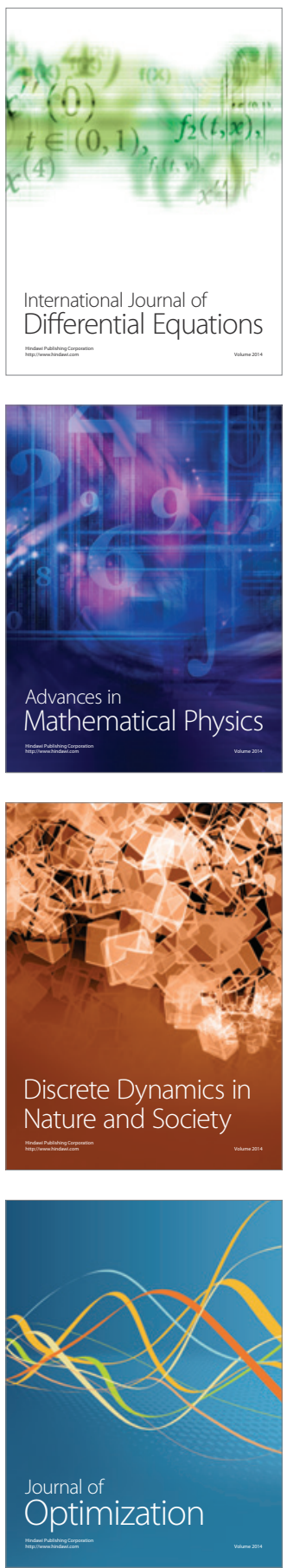